\title{
Drying and Impregnation for Heavy Timbers of Sugi Heartwood III - Preservative Penetration Observed on Boxed-Heart Square Timber of Sugi, Dried with Either The High-Temperature Setting Treatment or The Medium-Temperature Drying Adding Radio-Frequency Heating ${ }^{\dagger}-$
}

by

\author{
Yasuhiro TERANISHI*, Haruko SAKAI**, Hajimu KAIMOTO**, Katsunori MASUDA**, \\ Yoko TANAKA**, Takafumi ITOH**, Ikuo MOMOHARA***, Shigeki YATA****, \\ Noboru FUJIMOTO*****, Akira MAKITA******, Tomomi SHIGEYAMA*******, \\ Akio YAMAGUCHI********, Daisuke TEZUKA********* and Yuzo FURUTA**********
}

For the development of manufacturing technology of boxed-heart Sugi timber with appropriate preservative treatment, we have been examining the effects of the drying method before impregnation on the quality of the products with preservative treatment. We used boxed-heart Sugi timber from Nara and Oita which were treated either by medium-temperature drying, by a combination of medium-temperature drying and radio-frequency heating, by a high-temperature setting drying, or by a combination of high-temperature setting drying and radio-frequency heating. We investigated the penetration of the timber preservatives as well as the dimensional change before and after the impregnation. As a result, we confirmed that there were parts with insufficient penetration in boxed-heart Sugi timber from the above prefectures which were dried by a high-temperature setting treatment. Moreover, there were some cases in which such timbers had lower solution uptake and penetration ratio of preservatives than those that were dried with medium-temperature. Also, back split was necessary in order to prevent surface checks during drying process after impregnation and curing process. Lastly, using radio-frequency heating in addition to medium-temperature for drying before impregnation showed a possibility of producing materials without poor penetration, reduced solution uptake or penetration ratio in short time.

Key words:

Sugi heartwood, Impregnation, Preservative penetration, High-temperature setting treatment, Radio-frequency heating

\section{Introduction}

In order to use Sugi as structural materials of residences public buildings for extended period of time, it is necessary to develop a method which appropriately produces boxed-heart square Sugi timbers with preservative treatments. To give the required durability to boxed-heart square Sugi timbers, the processes such as drying after lumbering (before impregnation), incising, compressed impregnation and drying afterward should be combined appropriately.

As drying methods for boxed-heart square Sugi timbers, medium-temperature drying (referred to as MT), in which the timbers were dried with temperature less than $100^{\circ} \mathrm{C}$ has been used so far. However, in late years a high-temperature setting drying (referred to as HT) is widely used. In this method, timbers are treated with temperature higher than $100^{\circ} \mathrm{C}$ with low humidity condition (referred to as the high-temperature setting treatment) in the initial drying, and this method is known to produce products with few surface checks even with boxed-heart square timbers without back splits in short time $^{1)}$. Also, a combination drying method with radio-frequency heating ${ }^{2)}$ under the same schedules as in HT (referred to as HF) is also starting to spread.

On the other hand, it has been pointed out that the solution uptake of preservatives is reduced in materials treated with temperature higher than $100^{\circ} \mathrm{C}$ or over extended drying compared to those treated under room temperature or temperature below $100^{\circ} \mathrm{C}^{3), 4)}$.

However, in recent HT for Sugi timbers, the high-temperature setting treatment is often done for about 24 hours before drying with temperature below $100^{\circ} \mathrm{C}$ in

\footnotetext{
+ Received Sep. 16, 2015 (C)2016 The Society of Materials Science, Japan

* Member: Nara Forest Research Institute., Kibi, Takatori, 635-0133, Japan ** Nara Forest Research Institute., Kibi, Tkatori, 635-0133, Japan *** Forestry and Forest Products Research Institute, Matsunosato, Tsukuba, 305-8687, Japan ***** Yokohama National University, Professor Emeritus. ***** Faculty of Agriculture Graduate Schools of Kyushu University, Higashi-ku, Fukuoka, 812-8581, Japan ******* Dainihon Wood-Preserving Co.,LTD., Minato-ku, Nagoya, 455-8680, Japan ******* Xyence Co.,LTD., Naganuma-chou, Isesaki, 372-0855, Japan ******** KOSHII \& Co.,LTD., Suminoe-ku, Osaka, 559-0026, Japan ********* Kanematsu-NNK Co.,LTD., Koutou-ku, Tokyo, 136-8622, Japan ********** Life and Environmental Sciences Course, Graduate School of Kyoto Prefectural University, Sakyo-ku, Kyoto, 606-8522, Japan
} 
order to reduce internal checks ${ }^{5}$. There are few reports that discuss the impregnation characteristics of preservatives on Sugi materials treated under such practical treatment schedules. For this reason, in the previous report we studied the solution uptake and penetration of boxed-heart Sugi timbers from Nara, without back splits, which were dried by HT or HF. The report showed that the Sugi materials treated by such methods had lower solution uptake than those dried naturally or by $\mathrm{MT}^{6}$. Moreover, we found that some materials without back splits which were treated either by HT or HF had surface checks and dimensional change during the treatment process after impregnation and the curing process ${ }^{6)}$.

These two problems require reconfirmation and investigations for solutions as they significantly affect the materials with preservative treatments. As for the solution uptake for Sugi heartwood prepared by different treatment methods, the variations in the amount and penetration differ, even for timbers of same species, depending on the production site ${ }^{7)}$. Based on this fact, it is necessary to carry out the same test for the materials produced in other regions in addition to Nara, and to continue to collect resources to grasp whether it is a common problem to Sugi which varies in the production site. It is also important to search for methods that require fewer days for treatment without reducing the solution uptake. One of the candidate methods is the combination of medium-temperature below $100^{\circ} \mathrm{C}$ and radio-frequency heating (referred to as MF) ${ }^{8)}$. MF uses radio-frequency heating to increase steam pressure inside the material, transferring water to the surface, and applies medium-temperature at the same time to evaporate water ${ }^{9)}$. This method is expected to dry Sugi materials in short time without using the high-temperature setting treatment.

As for the problem of surface checks during drying process after impregnation and curing process, it is possible to reduce checks by applying back splits to the square timbers before impregnation ${ }^{10)}$. It is important to understand the dimensional change of the back splits after impregnation in order to set an appropriate oversizing for the surface carving before completing as final products.

In this study, we carried out $\mathrm{HT}, \mathrm{HF}$ and $\mathrm{MF}$, as drying method before impregnation, on boxed-heart square Sugi timbers from Nara and Oita with back splits. We recorded the numbers of days required to dry, investigated the solution uptake, penetration ratio, and dimensional change after the impregnation, and compared them to those with MT.

\section{Experiment}

In order to study the effect of the pre-impregnation drying method on the penetration, we used 9 boxed-heart square Sugi timbers from Nara and 9 from Oita (species: Yabukuguri). The cross-sectional dimension of each piece of material was $165 \times 165 \mathrm{~mm}$. This is for the final dimension of $150 \times 150 \mathrm{~mm}$, which is for low building of public. The length was $4 \mathrm{~m}$. We used boxed timbers most of whose part is heartwood. This is because drying and impregnation are both more difficult for heartwood than for sapwood ${ }^{7)}$. This way, the results to be obtained can then be applied to all Sugi materials regardless of the sapwood-heartwood ratio.

The impregnation preservative was copper-azole compound wood preservative (CUAZ). The preservative was diluted with tap water to make K3 work solution defined by Japanese Agricultural Standards (JAS) ${ }^{11)}$.

\subsection{Methods}

2.2.1 Preparation of test materials We used 9 boxed-heart square Sugi timbers from Nara and Oita. As shown in Fig. 1, 4 specimens of length $750 \mathrm{~mm}$ in the fiber direction were collected. We also obtained samples of length $20 \mathrm{~mm}$ in the fiber direction at locations a - e (Fig. 1) and measured the moisture content of each sample by oven-dry method. We measured the initial moisture content of each specimen as the average of the two surrounding samples. We estimated the oven-dried mass of each sample from its initial moisture content and mass. We sealed both ends of the specimens with epoxy resin bond immediately after collecting them.

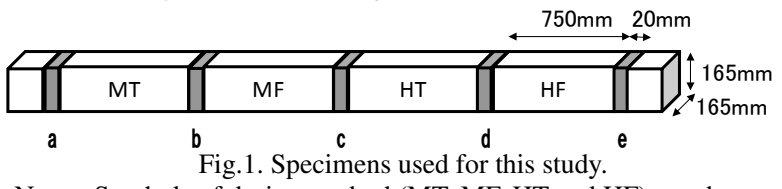

Notes: Symbols of drying method (MT, MF, HT and HF) are shown in Table 1., a-e : Specimens to estimate measure the initial moisture contents.

2.2.2 Drying before preservative impregnation For the four specimens collected from a same square timber, we used different methods until the moisture content fell below $20 \%$. The methods used in this study were MT, MF, HT and HF. As investigated in the previous study ${ }^{8)}$, the MF method can dry large section of Sugi material in short time without high-temperature setting treatment. Table 1 shows the drying conditions of each method. In order to sample a specimen for each drying method from each square timber of length $4 \mathrm{~m}$, we divided one timber into sections shown in Fig. 1. We applied a back split of width $3 \mathrm{~mm}$ and depth $82 \mathrm{~mm}$ on each specimen. Also, the HT and HF methods use high-temperature setting treatment which reduces surface checks, so they are commonly used for boxed-heart timbers without back splits. As we reported in a previous study), however, there are 
possibilities of surface checks on timbers, which are treated by HT or HF without back splits, during the drying process after impregnation or curing process. It is necessary to avoid checks after impregnation since unpreserved parts would be exposed to the surface. Therefore, we applied back splits to the specimens for the HT and HF methods in this study.

We occasionally measured the mass of each specimen during the drying process. We calculated the moisture content from this mass and the oven-dried mass collected in 2.2.1 and ended the process when the estimated moisture content reached below $20 \%$.

After dying, we adjusted the specimen's cross section to $154 \times 154 \mathrm{~mm}$. This dimension was chosen for cutting the materials after impregnation and drying into a wood cross section of $150 \times 150 \mathrm{~mm}$ as completing the product.

\begin{tabular}{|c|c|c|c|c|c|}
\hline $\begin{array}{c}\text { Symbols of } \\
\text { drying method }\end{array}$ & $\begin{array}{c}\text { Number of } \\
\text { specimens } \\
\text { (From Nara pref.) } \\
\text { (From Oita pref.) }\end{array}$ & $\begin{array}{c}\text { Initial } \\
\text { steaming } \\
\text { (D.B.T.a)/ } \\
\left.\text { W.B.T. }{ }^{\text {b) }}\right) \\
\text { (Time) } \\
\end{array}$ & $\rightarrow$ & $\begin{array}{c}\text { High-temperature } \\
\text { setting treatment } \\
\text { (D.B.T. } \\
\text { W.B.T. } / \\
\text { (Time) } \\
\text { (Time }) \\
\end{array}$ & 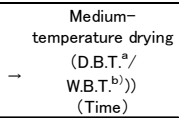 \\
\hline $\mathrm{MT}^{\mathrm{c})}$ & $\begin{array}{l}9 \\
9\end{array}$ & $\begin{array}{c}80^{\circ} \mathrm{C} / 80^{\circ} \mathrm{C} \\
12 \mathrm{hr}\end{array}$ & $\rightarrow$ & - & $\rightarrow \begin{array}{c}80^{\circ} \mathrm{C} / 77 \sim 70^{\circ} \mathrm{C} \\
466 \mathrm{hr}\end{array}$ \\
\hline $\mathrm{MF}^{\mathrm{d})}$ & $\begin{array}{l}9 \\
9\end{array}$ & $\begin{array}{c}80^{\circ} \mathrm{C} / 80^{\circ} \mathrm{C} \\
12 \mathrm{hr}\end{array}$ & $\rightarrow$ & - & $\begin{array}{c}80^{\circ} \mathrm{C} / 77 \sim 70^{\circ} \mathrm{C} \\
\rightarrow \quad 350 \mathrm{hr} \\
\quad \text { with radio- } \\
\text { frequency heating } \\
\end{array}$ \\
\hline$H T^{e)}$ & $\begin{array}{l}9 \\
9\end{array}$ & $\begin{array}{c}90^{\circ} \mathrm{C} / 90^{\circ} \mathrm{C} \\
12 \mathrm{hr}\end{array}$ & $\rightarrow$ & $\begin{array}{c}120^{\circ} \mathrm{C} / 90^{\circ} \mathrm{C} \\
24 \mathrm{hr}\end{array}$ & $\begin{array}{c}90^{\circ} \mathrm{C} / 60^{\circ} \mathrm{C} \\
72 \mathrm{hr}\end{array}$ \\
\hline $\mathrm{HF}^{\mathrm{f}}{ }^{\text {) }}$ & $\begin{array}{l}9 \\
9\end{array}$ & $\begin{array}{c}90^{\circ} \mathrm{C} / 90^{\circ} \mathrm{C} \\
12 \mathrm{hr}\end{array}$ & $\rightarrow$ & $\begin{array}{c}120^{\circ} \mathrm{C} / 90^{\circ} \mathrm{C} \\
24 \mathrm{hr}\end{array}$ & $\begin{array}{c}90^{\circ} \mathrm{C} / 60^{\circ} \mathrm{C} \\
\rightarrow \quad 72 \mathrm{hr} \\
\quad \text { with radio- } \\
\text { frequency heating }\end{array}$ \\
\hline
\end{tabular}

Notes: ${ }^{\text {a)}}$ : Dry-bulb temperature, ${ }^{\text {b)}}$ : Wet-bulb temperature, ${ }^{\text {c) }}$ : Medium-temperature drying, ${ }^{\text {d) }}$ : Medium-temperature drying adding radio-frequency heating, e): High-temperature setting drying, f). High-temperature setting drying adding radio-frequency heating.

2.2.3 Incising Upon compressed impregnation of the preservative into boxed-heart Sugi timbers, incising is effective for satisfying the standards of preservative penetration defined by $\mathrm{JAS}^{11)}$. In this report, we applied incising of depth $10 \mathrm{~mm}$, length $15 \mathrm{~mm}$ and density of $4000 / \mathrm{m}^{2}$ on the specimens after the drying before impregnation (with adjusted dimensions).

2.2.4 Impregnation of wood preservative We measured the masses $\left(W_{\mathrm{b}}\right)$, lengths in the fiber direction, and the width perpendicular to the fiber direction in each of the four side $\left(d_{\mathrm{b} 0}\right.$ for a side with back split, and $d_{\mathrm{b} 1}, d_{\mathrm{b} 2}, d_{\mathrm{b} 3}$ for the other sides without back split) of the specimens, and impregnated the CUAZ work solution to them by compression. The impregnation schedule included immersing the each specimen in the working solution, 30 minutes of reduced pressure at $50 \mathrm{hPa}$ followed by 2 hours of compression at $1.3 \mathrm{MPa}$. After impregnation, the specimens were removed from the work solution and left for about 1 hour until the dripping stopped, and the masses $\left(W_{\mathrm{a}}\right)$ were measured. The solution uptake $(S)$ was defined as follows,

$$
S=\frac{W_{\mathrm{a}}-W_{\mathrm{b}}}{V_{\mathrm{b}}},
$$

where $V_{\mathrm{b}}$ is the volume before impregnation.

2.2.5 Drying after preservative impregnation Drying after impregnation requires control so as to avoid changes in the impregnated preservative. In this study, we dried the specimens after impregnation through a common, medium-temperature schedule with dry-bulb temperature $60^{\circ} \mathrm{C}$ and wet-bulb temperature $55 \sim 45^{\circ} \mathrm{C}$. The drying period was about 6 days. After re-drying, we measured the widths $d_{\mathrm{r} 0}$ with back split and $d_{\mathrm{r} 1}, d_{\mathrm{r} 2}, d_{\mathrm{r} 3}$ without back split, which correspond to the width before the impregnation. The rates of dimensional changes after re-drying for a side with back split $\left(D_{\mathrm{r} 0}\right)$ and other sides without back split $\left(D_{\mathrm{r}}\right)$ were obtained using the following equations.

$$
\begin{aligned}
& D_{\mathrm{r} 0}=\frac{d_{\mathrm{r} 0}-d_{\mathrm{b} 0}}{d_{\mathrm{b} 0}} \times 100, \\
& D_{\mathrm{r}}=\frac{1}{3} \sum_{i=1}^{3} \frac{d_{\mathrm{r} i}-d_{\mathrm{b} i}}{d_{\mathrm{b} i}} \times 100 .
\end{aligned}
$$

After this process, we cured the specimens in a room for about 2 months.

2.2.6 Measuring the dimensions after conditioning and preservative penetration After two months of curing, we collected two samples of length $200 \mathrm{~mm}$ in the fiber direction from each specimen, avoiding knots and the parts within $50 \mathrm{~mm}$ from the edges. We placed those samples in a thermo-hygrostat with dry-bulb temperature $30^{\circ} \mathrm{C}$ and wet-bulb temperature $26.5^{\circ} \mathrm{C}$ (relative humidity $76 \%$ ), and conditioned the samples up to moisture content $15 \%$. The conditioning lasted for about 6 days, and after confirming the equilibrium of the masses, we measured the widths $d_{\mathrm{c} 0}$ with back split and $d_{\mathrm{c} 1}, d_{\mathrm{c} 2}, d_{\mathrm{c} 3}$ without back split, which correspond to the width before the impregnation. The rates of dimensional changes after conditioning for a side with back split $\left(D_{\mathrm{c} 0}\right)$ and other sides without back split $\left(D_{\mathrm{c}}\right)$ were obtained using the following equations.

$$
\begin{aligned}
& D_{\mathrm{c} 0}=\frac{d_{\mathrm{c} 0}-d_{\mathrm{b} 0}}{d_{\mathrm{b} 0}} \times 100, \\
& D_{\mathrm{c}}=\frac{1}{3} \sum_{i=1}^{3} \frac{d_{\mathrm{c} i}-d_{\mathrm{b} i}}{d_{\mathrm{b} i}} \times 100 .
\end{aligned}
$$

We sprayed chromazurol $\mathrm{S}$ solution on the cross sections of the other sample to color the parts penetrated by preservatives ${ }^{11)}$. In this study, similar to a previous report $^{6)}$, we prepared the cross section at $154 \times 154 \mathrm{~mm}$ for the final dimension of $150 \times 150 \mathrm{~mm}$. For this reason, we measured the penetration ratio in the interior up to $10 \mathrm{~mm}$ inside the final outer surfaces. The penetration ratio $(P)$ was calculated as follows,

$$
P=\frac{A_{\mathrm{c}}}{A} \times 100
$$

where $A$ is the cross-sectional area in the interior up to 10 
mm inside the final outer surfaces, and $A_{\mathrm{c}}$ is the area of colored part in the area $A$.

If $P$ was $80 \%$, we considered it satisfactory for the standard of K3 section defined by JAS.

\section{Results and observations}

3.1 Moisture content before preservative impregnation and penetration of preservative

Table 2 shows the moisture contents of the specimens dried by four different methods before impregnation. In each condition, the moisture content of each specimen as a whole was adjusted below $20 \%$ as intended. As for the distribution of moisture content, drying reach into the interior in each method. In the region from $0 \mathrm{~mm}$ to 10 $\mathrm{mm}$ from the outer surface, the moisture contents were below 20\% for most specimens. Fig. 2 shows the penetration status of the preservative. This figure uses material No. 42 (from Nara) and No. 94 (from Oita) to show the results of the specimens collected continuously in the fiber direction. Fig. 2 implied that, regardless of the production site, the preservative penetrated most of the region within $0 \mathrm{~mm}$ to $10 \mathrm{~mm}$ from the outer surface in MT and MF methods. On the other hand, in the HT and HF methods, there were some parts without coloration in the $0-10 \mathrm{~mm}$ region, as indicated by white arrows, where the penetration was not complete. As for the penetration from the back splits, the HT and HF methods led to smaller penetrated regions than the MT and MF methods. Previous reports ${ }^{6), 12)}$, in which specimens were collected in Nara, inferred that the preservative penetrates from the back splits and incised cuts and through bordered pits, which exist in the radiant wall of the early wood tracheid, and advances in the direction of the tangential lines. The reports also indicated that, with the high-temperature setting treatment, such penetration channels could close or shrink, leading to materials with unpenetrated parts. In this investigation, insufficient penetration seemingly due to the same cause was confirmed for materials from the same production site as well as another site. The above results pertain to materials No. 42 (from Nara) and No. 94 (from Oita), but similar tendency was observed in other specimens. However, there were some specimens from Oita, treated by the HT and HF methods, which had surface checks (Fig.2, HT No.94). There were 4 out of 9 specimens with surface checks for the HT method, and 3 out of 9 for the HF method. Such specimens, as shown in Fig. 3, had preservative penetration through the checks.

\subsection{Differences in the solution uptake and penetration ratio between drying methods}

Figure 3 shows solution uptake $(S)$ for all the specimens from the 9 bodies from Nara and 9 bodies from Oita. In order to compare $\mathrm{S}$ with respect to the test
Table 2. Moisture content of the specimens dried for impregnation.

\begin{tabular}{|c|c|c|c|c|c|c|}
\hline \multirow{3}{*}{ Symbol } & \multicolumn{6}{|c|}{ Moisture content(\%) } \\
\hline & \multicolumn{3}{|c|}{ From Nara pref. } & \multicolumn{3}{|c|}{ From Oita pref. } \\
\hline & Min. & Ave. & Max. & Min. & Ave. & Max. \\
\hline MT & 14.3 & 16.8 & 18.8 & 16.4 & 17.8 & 19.6 \\
\hline MF & 13.7 & 16.3 & 21.8 & 14.9 & 17.1 & 18.6 \\
\hline HT & 9.6 & 14.7 & 23.7 & 14.8 & 17.2 & 20.7 \\
\hline $\mathrm{HF}$ & 12.4 & 15.1 & 17.7 & 11.9 & 16.5 & 19.1 \\
\hline
\end{tabular}

Notes: Symbols of drying method are shown in Table 1.
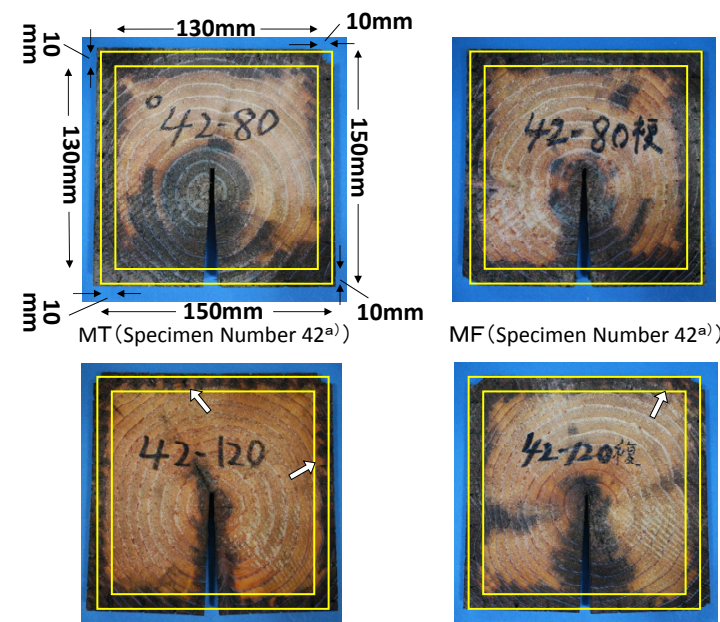

MF (Specimen Number $42^{a)}$ )
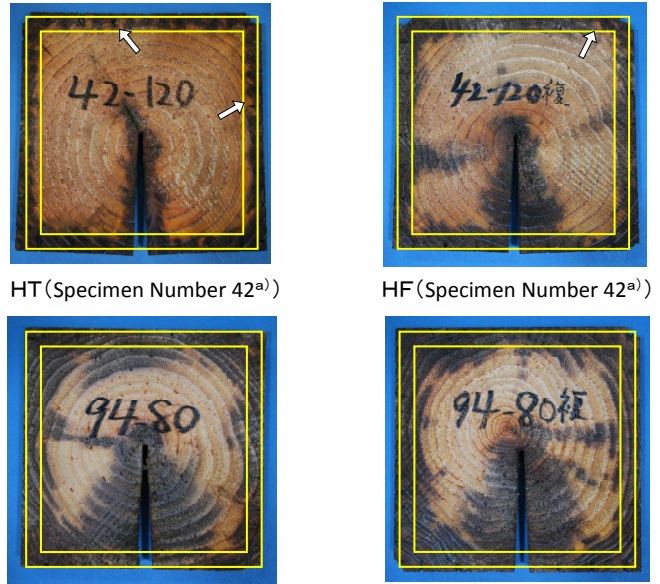

MT (Specimen Number 94b) Surface check

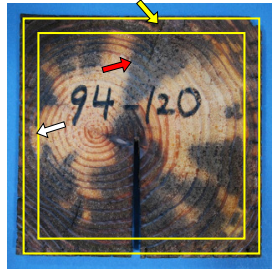

HT (Specimen Number 94 ${ }^{\text {b) }}$ ) $\mathrm{HF}$ (Specimen Number 42a)

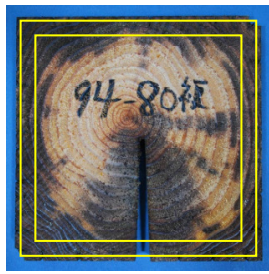

MF (Specimen Number 94b)

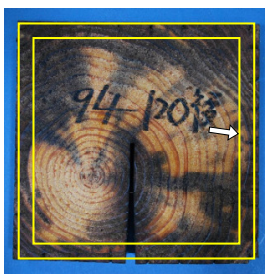

HF (Specimen Number 94b)

Fig.2. Examples of penetration patterns observed for each drying method in specimens cut from specimen number 42 and 94. Notes: Symbols of drying method are shown in Table 1. ${ }^{\text {a) }}$ : From Nara pref., ${ }^{\text {b) }}$ : From Oita pref.. White arrows indicate the example of the parts not penetrated. Red arrow indicates the example of the part penetrated from surface checks indicated by yellow arrow.

condition, Fig. 3 also shows the results of multiple comparisons. For the materials from Nara, the average $S$ was highest in the MT method, followed by MF, HT, and HF methods. $S$ by the HT and HF methods were significantly lower than that by the MT method at the $1 \%$ level of Tukey. This result matched the previous report ${ }^{6)}$. As for the materials from Oita, the average $S$ was highest in the MT method, as was the case for the materials from Nara. The average $S$ by the MF, HT, and HF methods were lower than that of the MT method, but there was no significant difference. From the fact that there were specimens from Oita treated by the HT and HF methods which had some part in the $0-10 \mathrm{~mm}$ region from the outer surface with insufficient penetration (Fig.2), it was 
unlikely that there had been no effect by the drying methods. We thus inferred that the absence of significant difference was due to the penetration through the surface checks made by the HT and HF methods. As a reference, the average $S$ after subtracting the estimated penetration through the surface checks was about $330 \mathrm{~kg} / \mathrm{m}^{3}$ for the HT method and $340 \mathrm{~kg} / \mathrm{m}^{3}$ for the HF method. These values were lower than the averages of the all specimens (HT: appr. $360 \mathrm{~kg} / \mathrm{m}^{3}$, HF: appr. $370 \mathrm{~kg} / \mathrm{m}^{3}$ ) shown in Fig. 3. Thus, it was confirmed once again that, for materials from Nara, the solution uptake tended to be lower for materials dried by the HT and HF methods before impregnation than those by the MT method. This reduced solution uptake was also possible for the materials from Oita, for which the penetrations through the surface checks could not be estimated.
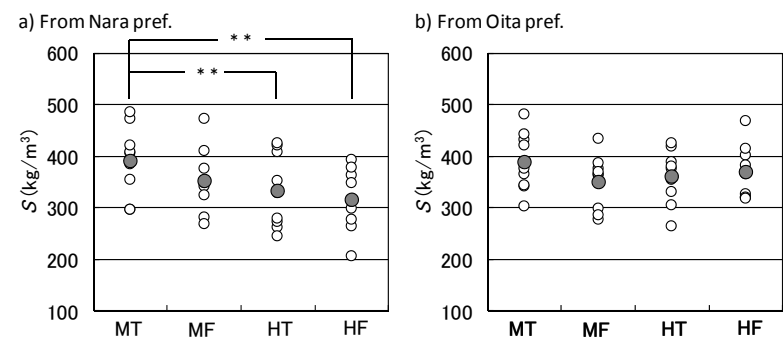

Fig.3. Effect of drying methods on solution uptake $S$.

Notes: $S$ is defined in section 2.2.4. Symbols of drying method are shown in Table 1. **: Significant difference at the $1 \%$ level of Tukey. Legend: : Average.
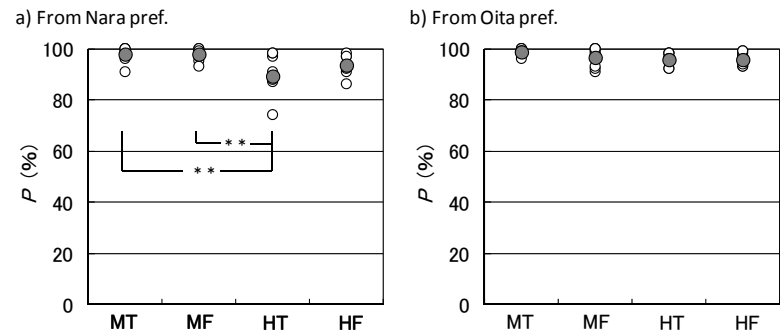

Fig.4. Effect of drying methods on penetration rate $P$. Notes: $P$ is defined in section 2.2.6. Symbols of drying method are shown in Table 1. **: Significant difference at the $1 \%$ level of Tukey. Legend: : Average.

Figure 4 shows penetration ratio $(P)$ for each specimen. Similar to the analysis of $S$, Fig. 4 also shows the results of multiple comparisons. First, for the materials from Nara, the average $P$ for the MT and MF methods was higher than those for the HT and HF methods. $P$ for the HT method was significantly lower than those for the MT and MF methods at the $1 \%$ level of Tukey. As for the materials from Oita, the average $P$ for the MT and MF methods was slightly higher than those for the HT and HF methods. However, there were no significant differences. As described above, this was likely due to the preservative penetration through the surface checks. As for $P$ of each specimen, most of the specimens from Nara reached the standard set by JAS, but material No. 42 (from Nara), shown in Fig. 2 and treated by the HT method, did not satisfy the criterion. As for the materials from Oita, all the specimens reached the criterion set by JAS.

Thus, there was a specimen treated by the HT method whose penetration ratio did not reach the JAS standard. However, the occurrence rate was not high; there was 1 out of 9 bodies in this study and 1 out of 12 bodies in the previous study $\left.{ }^{6}\right) 12$. Compared to the MT method, the HT method takes shorter time and is superior from the productivity point of view, and it is widely used in many plants. The raw material of preservative treatment is also easily available, so it is not necessarily reasonable to avoid the HT method from the results obtained in this study. However, in order to obtain boxed-heart Sugi timbers with appropriate treatment, a higher quality management seemed necessary in the production process. For example, as shown in Fig. 5, low solution uptakes $(S)$ tend to correspond to low penetration ratios $(P)$, indicating the possibility of estimating the penetration ratio from the solution uptake. Also, it could also be possible to increase the incising density in order to obtain the prescribed penetration ratio (JAS standard ${ }^{11)}$ ).

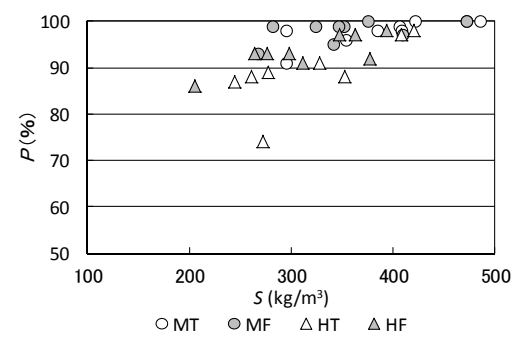

Fig.5. Relationship between solution uptake $(S)$ and penetration ratio $(P)$ observed on the specimens from Nara pref..

Note: $S$ and $P$ are shown in section 2.2 .4 and 2.2.6 respectively. Symbols of drying method are shown in Table 1.

\subsection{Change in dimensions after the preservative impregnation}

Figure 6 shows the rates of dimensional changes after re-drying $\left(D_{\mathrm{r} 0}, D_{\mathrm{r}}\right)$, and the rates of dimensional changes after conditioning $\left(D_{\mathrm{c} 0}, D_{\mathrm{c}}\right)$. There was almost no change in dimensions on sides without back splits $\left(D_{\mathrm{r}}\right)$ regardless of the test condition. On the other hand, the change in the dimension on sides with back splits $\left(D_{\mathrm{r} 0}\right)$ occurred for every test condition. Based on the fact that there were no surface checks formed after impregnation in any test condition, it was inferred that the dimensions changed because the back splits opened due to the difference in the moisture content before the impregnation and after the re-drying. Compared to the moisture contents before impregnation (Table 2), the average moisture content of the specimens after the re-drying was higher and was $23 \%$ for the MT method, $19.9 \%$ for the MF method, $22.9 \%$ for the HT method, and $21.1 \%$ for the HF method. Thus, it was likely that these specimens had open back 
splits after the treatment. On the other hand, the moisture content decreased to about $15 \%$ by curing and conditioning after the re-drying. $D_{\mathrm{c} 0}$ was smaller than that $D_{\mathrm{r} 0}$. This is likely to the decrease in the width of back splits. The above results were for the materials from Nara, but similar tendencies were found in the materials from Oita.

Thus, it was shown that it was possible to prevent new surface checks during impregnation and re-drying even for the materials treated by high-temperature setting treatment before impregnation by applying back splits at the beginning. On the other hand, there were large dimensional changes on the sides with back splits during the re-drying and curing processes. Thus, it should be noted that there can be dimensional changes, after shipping the products, on the sides with back splits if the final surface cut is done without appropriate control of moisture content in such processes.
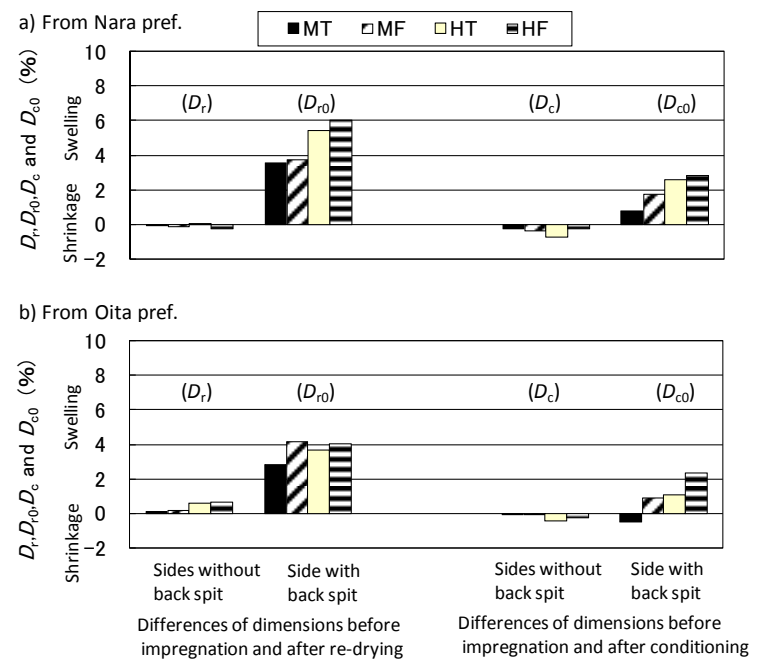

Fig.6. Effect of drying methods on dimensional changes for each step with and without back splits $\left(D_{\mathrm{r}}, D_{\mathrm{r} 0}, D_{\mathrm{c}}\right.$ and $\left.D_{\mathrm{co}}\right)$.

Notes: $D_{\mathrm{r}}, D_{\mathrm{r} 0}, D_{\mathrm{c}}$ and $D_{\mathrm{co}}$ are defined in section 2.2.5 and 2.2.6 respectively. Symbols of drying method are shown in Table 1. Re-drying methods and conditioning methods are shown in section 2.2 .5 and 2.2.6 respectively.

\subsection{Number of days required for drying before} impregnation

Figure 7 shows the specimens' moisture contents in the drying process by 4 methods before impregnation. Similar to Fig. 2, Fig. 7 shows the results for material No. 42 (from Nara) and material No. 94 (from Oita). According to Fig. 7, the decrease in moisture content was fast for the HT and HF methods, and it reached around $20 \%$ in about 4 days for specimens from both Nara and Oita. Also, the moisture content drop in the MF method was almost the same or slightly gentler, and the moisture content reached $20 \%$ in 4 days for materials from Nara and 5 days for materials from Oita. For the MT method, on the other hand, it took 10 days and 12 days to dry materials from Nara and Oita, respectively.
Figure 8 shows the relationship between the moisture content before the drying and the number of days required for the moisture content to reach $20 \%$ for each specimen. For the results for the materials from Nara, there was variety in required days depending on the moisture content before the drying, but the MF method took shorter days than the MT method for any moisture content before the drying. Also, the required days of the MF method were almost same as those of the HT and HF methods. The above findings were for the materials from Nara, but similar tendencies were found in the materials from Oita.
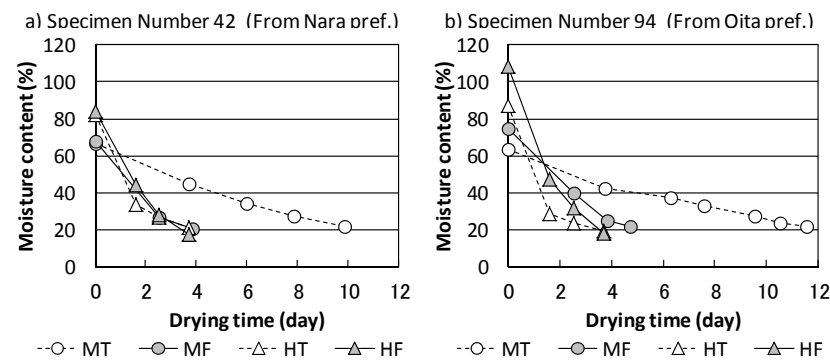

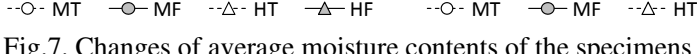
(Specimen Number 42 and 94) during the drying processes before impregnation.

Note: Symbols of drying method are shown in Table 1.
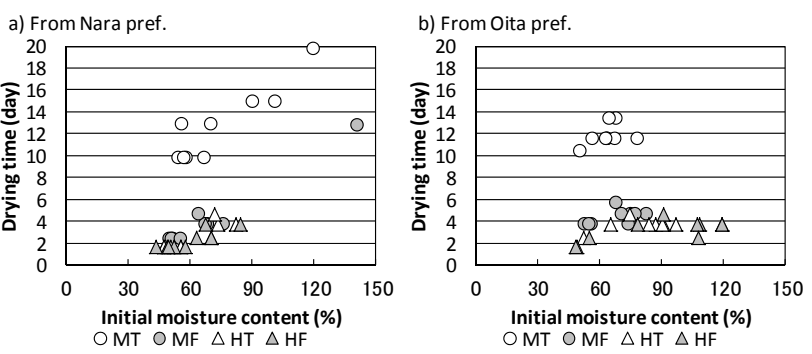

Fig.8. Relationship between initial moisture contents of green specimens and the times for kiln-drying to reduce moisture content below $20 \%$

Note: Symbols of drying method are shown in Table 1.

It was thus shown that it was possible to dry boxed-heart Sugi timbers in same period of time required for the HT and HF methods by using the MF method in the drying process before preservative impregnation. According to previous studies, though the HT and HF methods have high energy costs, the operation cycle becomes shorter due to the shorter time required for drying, and thus such methods can reduce the drying cost including the facility fees and labors ${ }^{13), 14)}$. Some calculations showed that those methods could lead to higher monthly production compared to other drying machines with same capacities ${ }^{13}$. We did not make cost calculations, but we showed that the MF method could dry boxed-heart Sugi timbers in same times as the HT and HF methods. Thus, the MF method can dry before preservative impregnation at lower costs than the MT method. Also, as shown above, in the MF method every specimen had the outer $0-10 \mathrm{~mm}$ region penetrated by 
the preservative, and its solution uptake and the penetration ratio were significantly different from those by the MT method. The MF, therefore, can be an effective drying method for the production of boxed-heart Sugi timbers with preservative treatment.

\section{Conclusion}

We used boxed-heart Sugi timbers from Nara and Oita with back splits, and applied HT, HF, and MF in the drying process before impregnation. We recorded the numbers of days required to dry, investigated the solution uptake, penetration ratio, and dimensional change after the impregnation, and compared them to those with MT. As a result, we obtained the following conclusions.

1. There were some parts with insufficient penetration in Sugi heartwood materials from Nara and Oita which were dried by HT or HF. It was suggested that the problem was common to Sugi which varies in the production site. Also, the solution uptake and penetration ratio by those methods were lower than those by MT. It was considered necessary to have quality management to meet the prescribed penetration ratio (JAS standard) when using such high-temperature methods for boxed-heart Sugi timbers with preservative treatment.

2. It was shown that it was possible to prevent surface checks during the impregnation and re-drying processes, even for the materials treated by HT or HF before impregnation, by applying back splits at the beginning. On the other hand, during the re-drying and curing process, it was considered necessary to manage the moisture content in order to prevent the change in dimensions of the sides with back splits.

3. It was shown that MF could dry materials before impregnation in same period of time required for HT or $\mathrm{HF}$ and without insufficient solution uptake or penetration ratio.

This study was done as part of the development /promotion projects of the new community materials for long-lasting quality residences under the "Comprehensive measures for the share expansion of community materials for residences in 2010" as a supplemental project for the Forestry Agency. We thank Mr. Minoru Yasujima from Yasujima Ltd. and Mr. Mitsuyuki Myojin from Yasujima Ltd. central research institute, who provided the machine and operations for incising.

\section{References}

1) T.Yoshida, T.Hashizume and N.Fujimoto,"High-temperature drying characteristics on boxed-heart square timber of Karamatsu and Sugi - influence of high temperature conditions with low humidity on drying properties-", Mokuzai Kogyo, Vol.55, No.8, pp.357-362(2000).

2) N.Kuroda, "Trends of wood drying research in Japan", Mokuzai
Gakkaishi, Vol.51, No.1, pp.10-12(2005).

3) H.Kurisaki and K.Mizumoto, "Kouon kansou sita sugi zai no yakuzai chunyu sei hyouka", Abstracts of the 51th Annual Meeting of the Japan Wood Research Society, p556(2001) The Japan Wood Research Society.

4) T.Ide, F.Sasaki, J.Ida, M.Ohkoshi and Y.Furuta, "Sugi zai no ekitai sintou sei ni oyobosu kansou ondo no eikyou - yuuen hekikou no heisa ritu tono kankei-", Abstracts of the 62th Annual Meeting of the Japan Wood Research Society, CD-ROM, C15-P-AM05 (2012) The Japan Wood Research Society.

5) T.Yoshida, T.Hashizume, T.Takeda, T.Tokumoto and A.Inde, "Reduction of surface checks by the high-temperature setting method on kiln drying of Sugi boxed-heart timber without back-splitting", Joural of The Society of Materials Science, Japan, Vol.53, No.4, pp.364-369(2004).

6) Y.Teranishi, H.Sakai, H.Kaimoto, K.Masuda, Y.Tanaka, T.Itoh, I.Momohara, S.Yata, N.Fujimoto, A.Makita, T.Shigeyama, A.Yamaguchi and D.Tezuka, "Drying and impregnation for heavy timbers of Sugi heartwood II - preservative penetration and surface checking observed on boxed-heart timber dried with a high-temperature setting method", Mokuzai Gakkaishi, Vol.60, No.3, pp.177-185(2014).

7) T.Shigeyama, "Mokuzai hozon gaku nyumon kaitei 3 han", pp.141-142(2012) Japan Wood Protection Association.

8) Y.Teranishi, K.Ono, R.Okinaka and T.Kubo, "The study of kiln drying system with combination of high-frequency heating and hot air heating( I ) - drying property and difference of heating efficiency affected by the thickness of stickers", Bulletin of the Nara Prefectural Forest Experiment Station, Vol.28, pp29-33(1998).

9) Y.Kawai, Y.Kobayashi, Y.Ishimaru and M.Norimoto, "Hybrid drying with high frequency heating and hot-air under atmospheric pressure $\mathrm{V}$ - combined effect of high frequency heating and hot air", Mokuzai Gakkaishi,Vol.49, No.6, pp.408-415(2003).

10) H.Kurisaki, Y.Myodo, N.Yonezawa and H.Taniguchi, "Sewari nado no hiware boushi kakou no hozon syori sugi marubou no taikyuusei eno kiyo", Abstracts of the 64th Annual Meeting of the Japan Wood Research Society, CD-ROM, N15-1015 (2009) The Japan Wood Research Society.

11) MAFF Notification 1920(2013) "Japanese agricultural standards for timber", Ministry of Agriculture, Forestry and Fisheries.

12) H.Sakai, Y.Teranishi, H.Kaimoto, K.Masuda, Y.Tanaka, T.Itoh, I.Momohara, S.Yata, N.Fujimoto, A.Makita, T.Shigeyama, A.Yamaguchi and D.Tezuka, "Drying and impregnation for heavy timbers of Sugi heartwood (1) - effects of moisture content and incising on preservative penetration", Mokuzai Hozon, Vol.37, No.1, pp.12-19(2011).

13) T.Hisada, "Wakariyasui kansou zai seisan gijyutsu manyuaru kaitei sin han", pp.92-93(2006) Japan Federation of Wood-industry Associations.

14) Y.Nakashima and Y.Iijima, "Cost analysis involving surface-check effects for various drying methods of Sugi square timbers", Mokuzai Kogyo, Vol.68, No.12, pp.570-575(2013). 